\title{
Estudio de los efectos hidráulicos aguas arriba de un dique de corrección torrencial
}

\author{
Pérez-Soba Diez del Corral, Ignacio ${ }^{1}$ \\ ${ }^{1}$ Doctor Ingeniero de Montes. Servicio Provincial de Agricultura, Ganadería y Medio Ambiente de Zaragoza. \\ Gobierno de Aragón. Paseo de María Agustín, 36. 50071 Zaragoza. \\ *e-mail: iperezs@aragon.es
}

\begin{abstract}
Resumen
Tradicionalmente, en los proyectos de diques transversales hidrológico-forestales se estudia el comportamiento de la corriente en la propia estructura y aguas abajo. Sin embargo, no debe olvidarse el efecto embalse, es decir, el frenado de la corriente aguas arriba, especialmente importante en los diques sitos en torrentes con caudal permanente en los que se produce siempre que discurra cualquier caudal mayor del que puede ser evacuado por los mechinales. Este efecto es deseable, porque hace que el agua progrese en condiciones mucho más favorables para la contención del fenómeno torrencial, por lo que procede en fase de proyecto comprobar que las dimensiones del dique son adecuadas para conseguirlo. La comunicación propone para ello determinar el nuevo régimen hidráulico del tramo según las alturas del agua en el paramento superior para cada caudal circulante, de acuerdo con la capacidad de desagüe del dique; y tras ello, estudiar los caudales máximos y mínimos que, circulando en esas condiciones de desagüe, mantienen en las distintas secciones una fuerza de arrastre superior a la límite. Este método determina entre qué valores de caudal subsiste el régimen torrencial aguas arriba del dique (denominado en la comunicación como FTR, fenómeno torrencial residual), y qué tipo de influencia puede transmitirse a través de él aguas abajo.
\end{abstract}

Palabras clave: Diques hidrológico-forestales. Torrentes. Sedimentos.Hidráulica 


\section{Introducción y objetivos}

La construcción de un dique transversal de corrección de ramblas o torrentes se hace con el evidente propósito de alterar el régimen hidráulico del tramo del cauce en el que se ubica, disminuyendo su torrencialidad. En los proyectos de construcción de diques siempre se realiza el estudio hidráulico en la propia estructura (para el correcto dimensionamiento de sus distintos elementos) y aguas abajo (para evitar los fenómenos de socavación o de agravamiento del régimen torrencial que pudieran producirse como consecuencia del obstáculo que para la corriente supone la presencia del dique). Pero, en lo referente a la torrencialidad aguas arriba, a menudo sólo se calcula la pendiente de compensación, es decir, la que se produce en la cuña de sedimentos retenidos por el dique, una vez esta cuña ha llegado hasta el umbral del vertedero del dique (Pérez-Soba, 2002).

Sin embargo, la vida útil del dique se puede dividir en tres fases (López Cadenas de Llano, dir., 1998):

- La primera, de colmatación progresiva.

- La segunda, durante e inmediatamente después del aterramiento.

- Y la tercera, cuando el lecho ya se ha consolidado. Sólo en las dos últimas se ha producido la pendiente de compensación; en la primera, el dique no está colmatado y limita la evacuación de agua en ese tramo a la capacidad de desagüe de las troneras o mechinales abiertos en el cuerpo del dique. Se produce por tanto aguas arriba el conocido "efecto embalse" o "efecto de presa", un frenado de la corriente que hace que ésta progrese en condiciones mucho más favorables para la contención del fenómeno torrencial: se eleva el calado, lo que conlleva el aumento de la sección mojada y por tanto la disminución de la velocidad del régimen y de la energía interna de la corriente.

De hecho, y dependiendo de los objetivos de la corrección y del tipo de torrente, puede que al Ingeniero de Montes proyectista interese prolongar lo máximo posible esta fase anterior al aterramiento. Para ello, es muy útil diseñar mechinales que no retengan todos o la gran mayoría de los materiales sólidos, sino que hagan una selección y dejen pasar la parte correspondiente a los tamaños y caudales que no impidan el objetivo general a toda corrección: la estabilización progresiva de un régimen fluvial aguas abajo. Esta selección se produce, en primer lugar, al limitar el paso de los que tengan mayor tamaño que los mechinales; y en segundo lugar, como consecuencia del efecto embalse antes descrito, ya que la disminución de la velocidad supone la correlativa de su capacidad de transporte de sólidos (Bureau of Reclamation, 2006).

Por tanto, procede en fase de proyecto comprobar que las características de desagüe del dique (en particular, el tamaño y la forma de los mechinales) cumplirán de manera suficiente los dos objetivos del efecto embalse durante el período anterior al aterramiento: la limitación del fenómeno torrencial aguas arriba, y la selección de la aportación de caudales sólidos aguas abajo. El objetivo de esta comunicación es proponer una metodología para ello, mediante métodos y fórmulas de hidráulica general. 


\section{Metodología}

Como se ha expresado, se usarán coeficientes y fórmulas de hidráulica general muy conocidas, disponibles en la bibliografía de referencia (García Nájera, Ripoll y Jiménez, 1959; García Nájera, 1962; López Cadenas de Llano, 1965; Chow, 1982; Mintegui y López Unzu, 1990; López Cadenas de Llano, dir., 1998; Jover y Vidania, 2001). El esquema de la metodología empleada, que es una aportación original de esta comunicación, se presenta en la Fig. 1 .

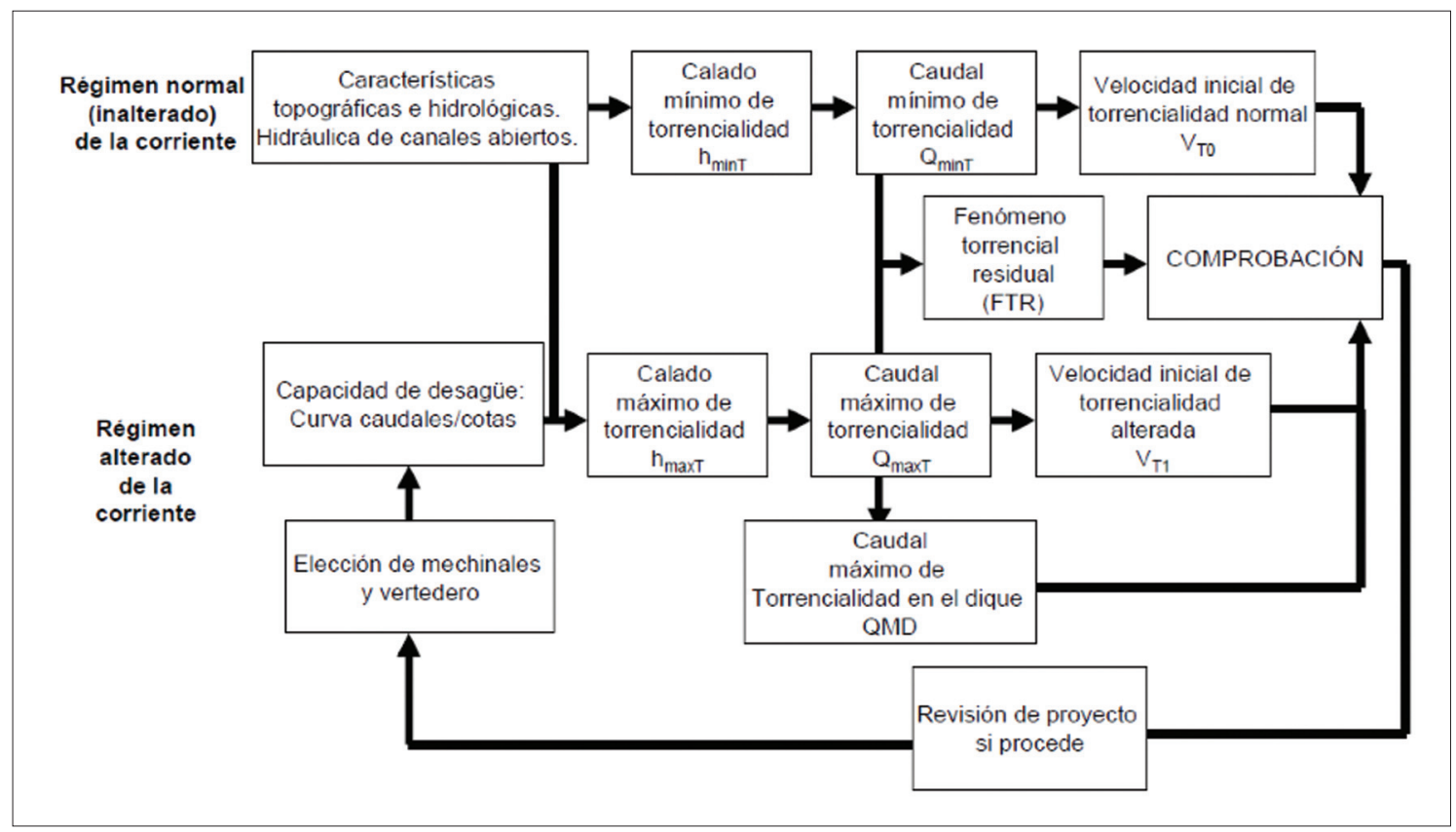

Figura 1. Metodología para el estudio de la torrencialidad aguas arriba de un dique.

Se calcula en primer lugar la capacidad de desagüe del dique. Para ello, lógicamente, hemos de hacer previamente el estudio y elección de dimensiones y tipo de los elementos de desagüe: tanto de los mechinales como del vertedero. El resultado de ese estudio se puede expresar mediante un gráfico de caudales circulantes frente a cotas de la lámina aguas arriba.

Ello nos permite, a su vez, calcular, para cada uno de los perfiles transversales aguas arriba, los valores de caudal (Q) y de calado (h) que establecen la separación entre los dos estados del régimen hidráulico con que circulará el agua como consecuencia de la presencia del dique: régimen "normal" o inalterado (el que seguiría la corriente en el cauce sin alterar, y que rige en todo el tramo no afectado por la lámina de agua que se crea por la presencia del dique) o alterado (en que sí lo es). Ello nos dará para cada perfil un caudal máximo y uno mínimo que, circulando en esas condiciones de desagüe, mantienen en esa sección una fuerza de arrastre superior a la límite. Del examen de esos caudales en relación con los propios del régimen normal de la corriente se pretende obtener conclusiones acerca del acierto en el diseño de los elementos de desagüe, y su mejora si procede en fase de proyecto. 
Para facilitar la comprensión de lo que se va exponiendo, se aplicará cada uno de los pasos a un caso concreto de dique ("dique-ejemplo"), para el que se ha elegido uno de $15 \mathrm{~m}$ de altura útil, vertedero de $5,3 \mathrm{~m}$ de altura y cuatro mechinales de superficie unitaria de $1,4 \mathrm{~m}^{2}$ (uno de fondo y tres intermedios), que se presenta en la Fig. 2, y que ya usamos en otros trabajos anteriores para el estudio de los efectos hidráulicos de un dique (Pérez-Soba, 2011). La elección de este ejemplo se basa en varias de sus cualidades:

1. Su notable altura útil asegura un notable efecto de presa.

2. El amplio tamaño de sus mechinales le otorga la consideración de dique semivacío, con amplia capacidad de desagüe.

3. La ubicación elegida para sus mechinales obliga a aplicar a cada uno de ellos distintas fórmulas para el cálculo de su capacidad de desagüe.

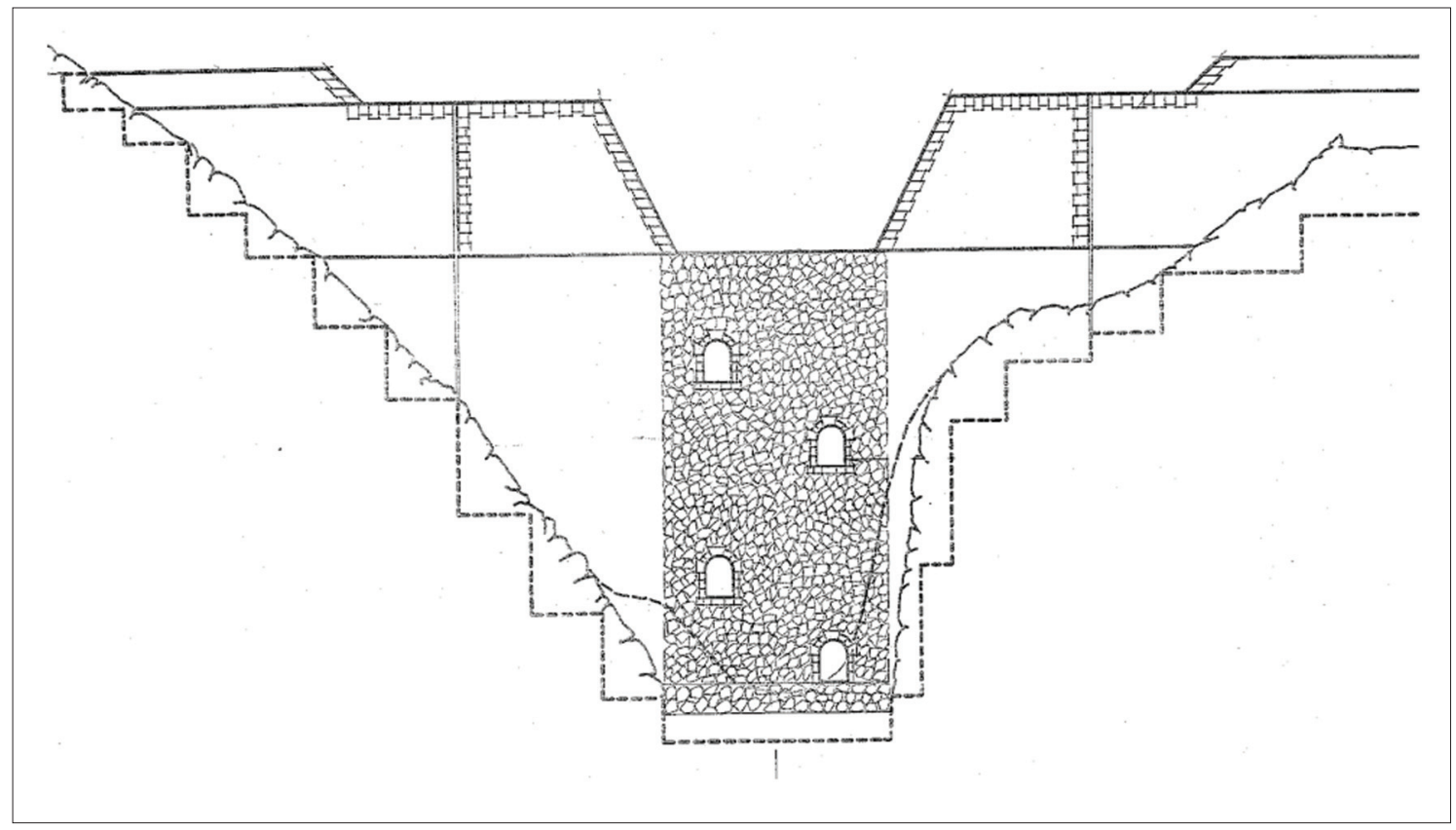

Figura 2. Dique-ejemplo usado para el cálculo.

En cuanto a los perfiles transversales y longitudinales del cauce, que se precisarán para el resto de los cálculos, se han tomado datos reales de un cauce torrencial.

\section{Resultados}

\subsection{Estado representativo del régimen hidráulico alterado mediante gráfico de caudales/cotas}

En lo que se refiere al cálculo de la capacidad de desagüe de los mechinales, ha de tenerse en cuenta que no es despreciable el rozamiento con las paredes, y que la fórmula a aplicar será muy distinta según la altura a la que se halle la lámina de agua 
con respecto al orificio. En régimen libre, el mechinal de fondo se puede calcular como vertedero practicado en pared de altura nula con contracción lateral de la lámina, mientras que los superiores se asimilarían a vertederos de pared gruesa con contracción lateral. En cambio, cuando están sumergidos son asimilables a un desagüe por orificio con tubo adicional, ya que la presión debida al gradiente hidráulico influye más que la velocidad con la que llega la corriente (Blein, 1947; Pérez-Soba, 2008). El resultado de ese estudio se puede expresar mediante un gráfico de caudales circulantes frente a cotas de la lámina aguas arriba, que permite el rápido cálculo de las cotas que alcanzan los caudales circulantes como resultado de la presencia del dique. En la Figura 3 se presenta la curva de cotas-caudales para el dique-ejemplo, en la cual se observa, evidentemente, un incremento muy brusco de la capacidad de desagüe en cuanto empieza a funcionar el vertedero.

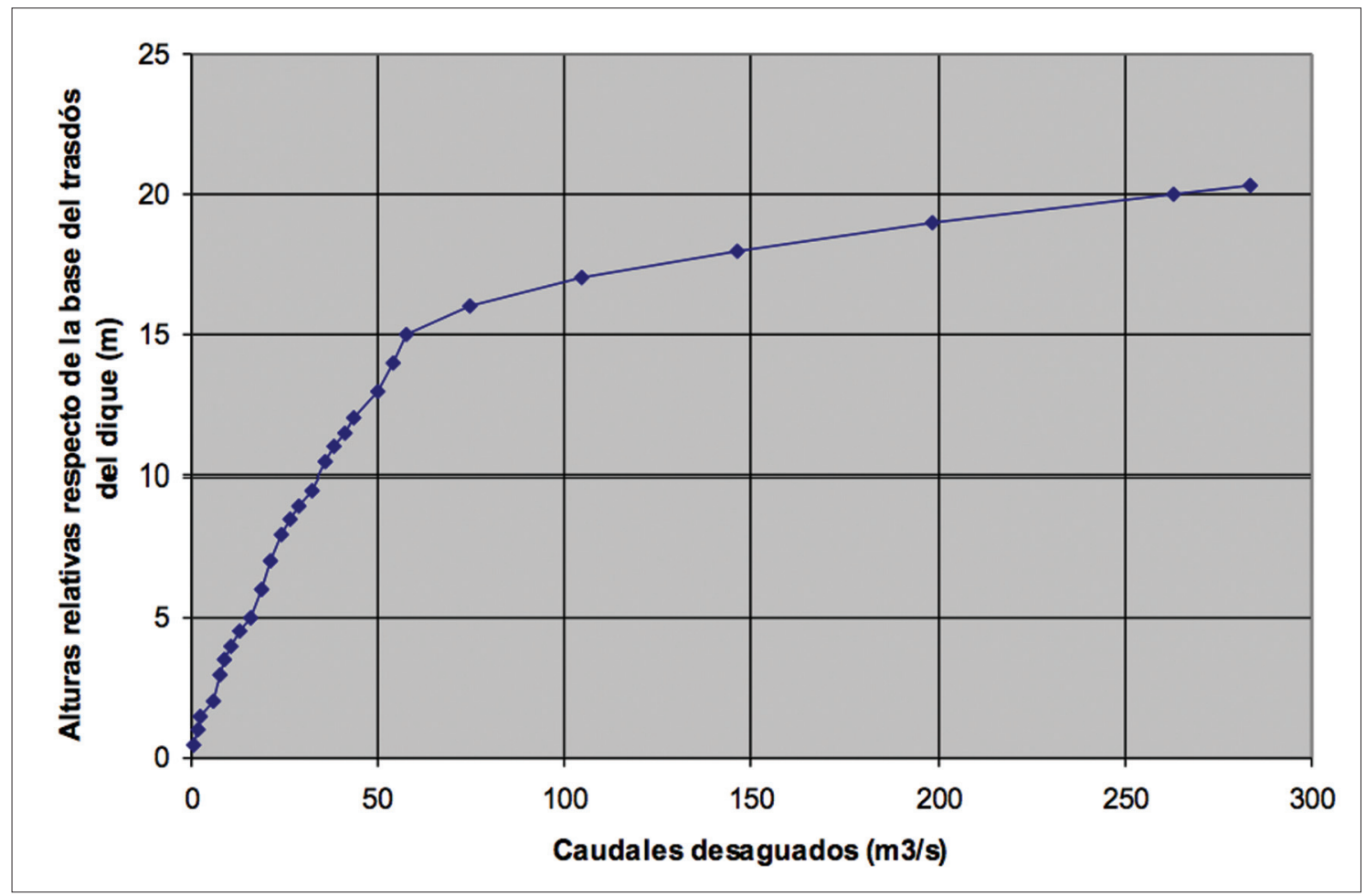

Figura 3. Curva de cotas-caudales para el dique ejemplo.

\subsection{Valores de caudal definitorios del fenómeno torrencial residual (FTR) aguas arriba}

La celeridad de la elevación o intumescencia provocada por un obstáculo (el dique, en este caso) es superior a la de la corriente, por lo cual se propaga aguas arriba, adoptando teóricamente la forma de una curva convexa, pero traduciéndose en los fenómenos de resalto en los cauces naturales (a causa de la absorción de energía debida a los torbellinos) en una simple ondulación, que se asimila virtualmente a la ho- 
rizontal (Chow, 1982). Por tanto, el estado representativo del régimen establecido aguas arriba es el formado por una superficie horizontal cuya altura se define para cada caudal de acuerdo con los valores obtenidos en el gráfico caudales-cotas antes establecido, y que se detendrá en el punto en que su calado se iguale con el calado conjugado igual al régimen normal o inalterado.

Por tanto, para calcular los valores de caudal y de calado que establecen la separación entre el régimen hidráulico normal y el alterado, bastará hallar para cada perfil Pi el calado para el que se iguala el caudal desaguado en régimen normal $\left(\mathrm{Q}_{\mathrm{i}}\right)$ y el desaguado por el dique según el gráfico caudales-cotas, para una cota respecto a la base del dique igual a la suma de la cota del lecho del perfil ( $\left.h_{\circ i}\right)$ más el calado "hi", tal como se refleja en la Figura 4. Para los valores menores de caudal o calado, el régimen hidráulico no resulta modificado y usaremos las formulaciones que corresponden al cauce inalterado; para valores superiores, el efecto embalse ya altera dicho régimen, y deberemos usar otras fórmulas y métodos; a este caudal podemos llamar caudal de alteración del régimen hidráulico (QARH). En la Figura 5 se presentan gráficamente, para el dique-ejemplo, los caudales y calados que, para cada uno de los perfiles transversales del cauce, determinan el tránsito del régimen hidráulico normal al alterado.

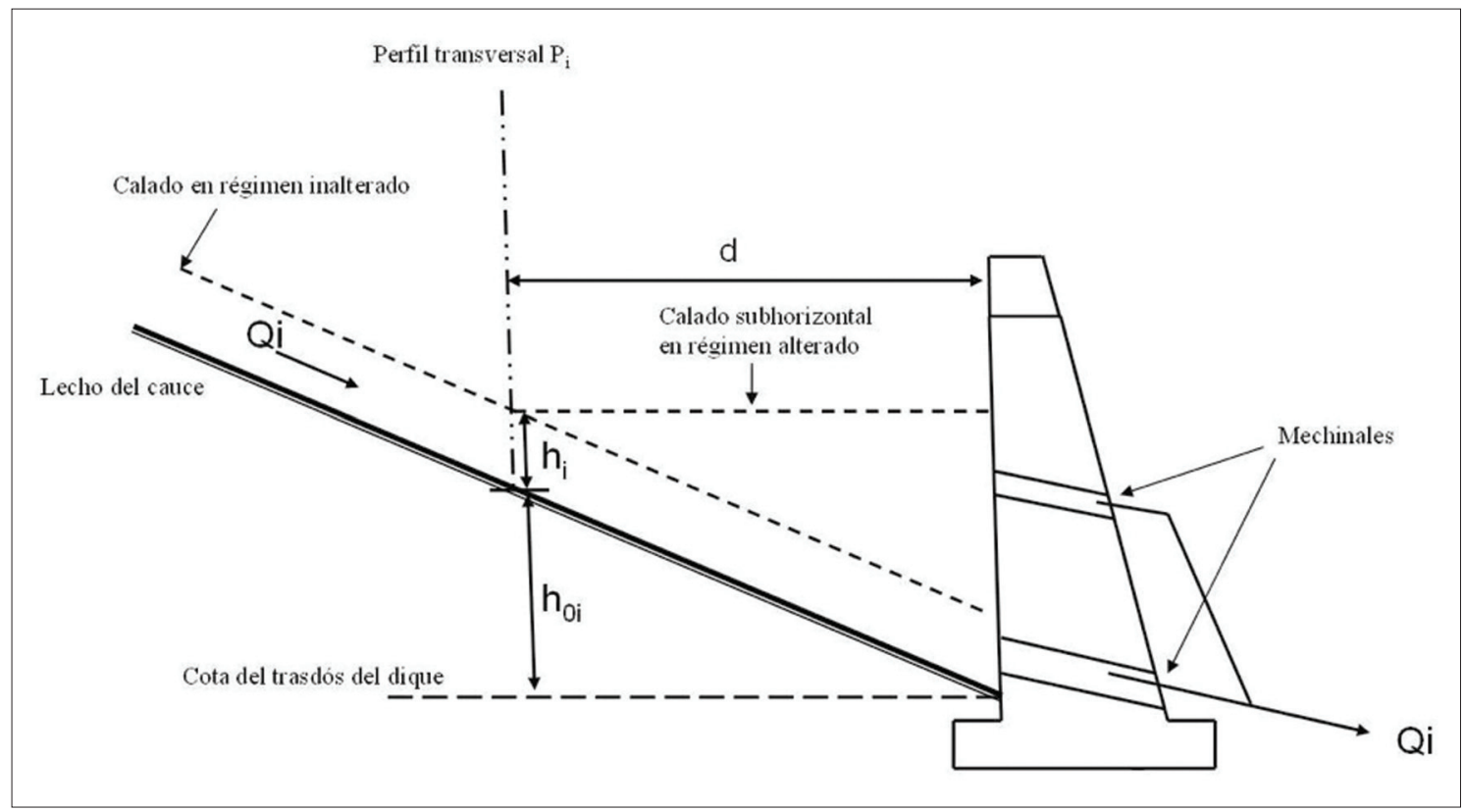

Figura 4. Esquema hidráulico de la situación límite entre el régimen hidráulico normal y el alterado para un perfil transversal Pi dado, aguas arriba del dique.

Por tanto, para cada perfil transversal aguas arriba del dique, el régimen sólo es torrencial entre dos valores. En primer lugar, un umbral inferior (que llamaremos caudal mínimo de torrencialidad, $\mathrm{Q}_{\min } \mathrm{T}$ ), correspondiente al caudal a partir del cual, en el régimen inalterado, comienza el estado torrencial, el cual se mantendría para todos los valores superiores si no existiera el dique. Pero la presencia de éste (y ne- 


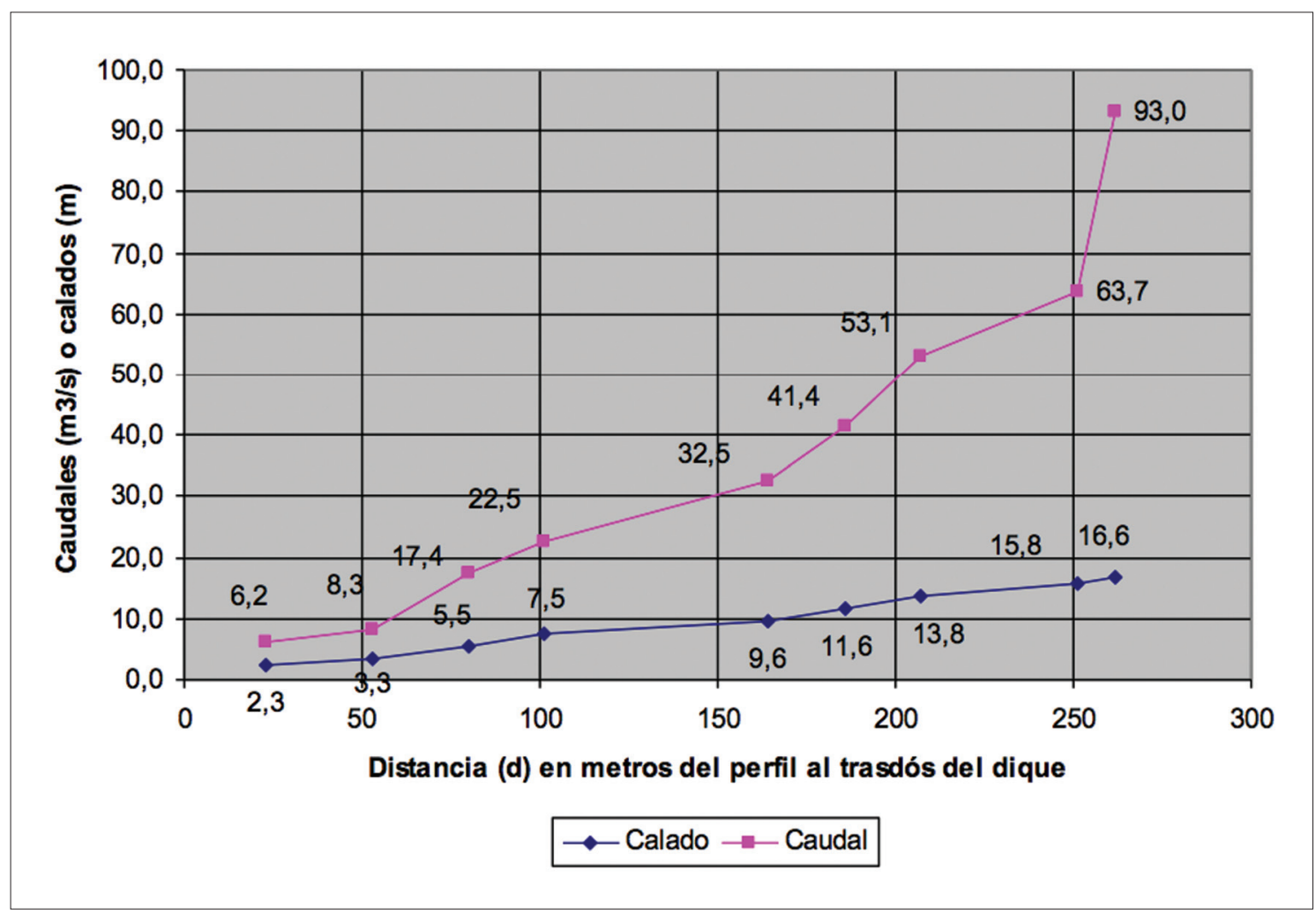

Figura 5. Valores del caudal de alteración del régimen hidráulico (QARH), y de sus calados correspondientes, para cada uno de los perfiles transversales del cauce, en el dique-ejemplo.

cesariamente, por tanto, dentro del régimen alterado) frena la corriente, de modo que existe un caudal (que llamaremos caudal máximo de torrencialidad, $\mathrm{Q}_{\max }$ ) a partir del cual desaparece la torrencialidad. Los valores $Q_{\min T}$ y $Q_{\max }$ acotarían por tanto un rango de caudales que podemos denominar "fenómeno torrencial residual" (FTR). No debe confundirse $\mathrm{Q}_{\max }$ con el valor de QARH antes expresado, ya que con valores superiores a QARH puede seguir existiendo torrencialidad; es decir: $\mathrm{Q}_{\min \mathrm{T}}<\mathrm{QARH}<$ $\mathrm{Q}_{\max }$.

Podemos calcular los dos umbrales del FTR aplicando la conocida fórmula de la tensión hidráulica de la corriente: $\mathrm{T}=\omega \cdot \gamma=\omega \cdot \mathrm{h} \cdot \mathrm{j}$, donde $\omega$ es el peso específico del agua; h el calado, $\mathrm{j}$ la pendiente y $\gamma$ el valor (en metros) de la tensión de arrastre, que habremos calculado para el tramo (en el ejemplo, $\gamma=0,0078 \mathrm{~m}$ ). Podemos prescindir en nuestros cálculos de $\omega$, ya que presentará valores casi idénticos para el régimen normal o el alterado. Por tanto, en el caso de $\mathrm{Q}_{\min \mathrm{T}}$ (régimen normal), la pendiente $\mathrm{j}$ será la del cauce, y por tanto:

$$
\boldsymbol{h}_{\min T}=\frac{\gamma}{j}
$$

A partir del valor de $h_{\min }$ (calado mínimo de torrencialidad) y por la función de caudales desaguados en régimen normal obtenido del apeo de cada perfil, se obtiene el valor de $\mathrm{Q}_{\min T}$. 
Sin embargo, como ya hemos dicho, el valor de $\mathrm{Q}_{\max \mathrm{T}}$ se producirá necesariamente dentro del régimen alterado, y por ello el valor de " $\mathrm{j}$ " no puede ser la pendiente real del cauce, sino la correspondiente a un cauce "virtual" que, en régimen normal y con el mismo calado que existe en el perfil, evacuara el mismo caudal. Ello nos obliga a calcular por iteraciones, de la siguiente forma: se toma un valor que se considere adecuado del calado (h) y se determina en la curva caudales/cotas el caudal Q correspondiente a la cota del lecho del perfil incrementada en h. A partir de allí se calcula la velocidad del agua a partir del dato de la superficie mojada para ese perfil ( $\mathrm{V}=$ $\mathrm{Q} / \mathrm{S}$ ), y finalmente (y una vez determinado el valor del radio hidráulico $\mathrm{R}$ ), se aplica la fórmula de la velocidad de Gauckler-Manning-Strickler:

$$
\boldsymbol{V}=\mathrm{n} \cdot \boldsymbol{R}^{2 / 3} \cdot j^{1 / 2} \Rightarrow j=\frac{\boldsymbol{V}^{2}}{n^{2} \cdot \boldsymbol{R}^{4 / 3}}
$$

El coeficiente de Strickler (n) está tabulado para distintas situaciones (Hager, 2010), siendo frecuente el uso de $n=30$ para cauces de gran rugosidad, como haremos para nuestro ejemplo. Así se determina el valor de j, con el cual se obtiene $\gamma=\mathrm{h} \cdot \mathrm{j}$, que se compara con el que se sabe correcto. Repitiendo el cálculo, se fija por interpolación el valor de $h_{\max }$ (calado máximo de torrencialidad) al que corresponderá un valor en la curva de caudales-cotas que será el $Q_{\max }$ buscado.

A partir de los valores de $Q_{\min T}$ y $Q_{\max T}$, a su vez, podemos calcular las velocidades de aguas limpias correspondientes a ambos caudales. Estas velocidades no pueden ser denominadas ni mínima ni máxima, pues no lo son, sino que las designaremos como velocidades iniciales de torrencialidad normal $\left(\mathrm{V}_{\mathrm{T} 0}\right)$ y de torrencialidad alterada $\left(\mathrm{V}_{\mathrm{T} 1}\right)$.

En la Tabla 1 se presentan, para cada uno de los perfiles transversales considerados para el dique-ejemplo, los valores de $h_{\min T}, h_{\max }, \mathrm{Q}_{\min }, \mathrm{Q}_{\max }, \mathrm{V}_{\text {т0}}, \mathrm{V}_{\mathrm{T} 1}$ у FTR calculados siguiendo la metodología expuesta.

Tabla 1. Resultados de la aplicación de la metodología propuesta al cálculo de los caudales torrenciales mínimo y máximo, para cada uno de los perfiles usados para el dique-ejemplo.

\begin{tabular}{|c|c|c|c|c|c|c|c|}
\hline \multirow{2}{*}{$\begin{array}{c}\text { Distancia } \\
\text { del perfil } \\
\text { al dique (m) }\end{array}$} & \multicolumn{3}{|c|}{ Régimen inalterado } & \multicolumn{3}{c|}{ Régimen alterado } & \multirow{2}{*}{ FTR } \\
\cline { 2 - 7 } & $\boldsymbol{h}_{\operatorname{minT}}$ & $\mathbf{Q}_{\operatorname{minT}}$ & $\mathbf{V}_{\text {TO }}$ & $\boldsymbol{h}_{\operatorname{maxT}}$ & $\mathbf{Q}_{\operatorname{maxT}}$ & $\mathbf{V}_{\text {T1 }}$ & \\
\hline 23 & 0,12 & 1,32 & 1,84 & 0,48 & 6,54 & 2,14 & 5,22 \\
53 & 0,14 & 2,32 & 1,84 & 0,41 & 8,64 & 2,20 & 6,32 \\
80 & 0,10 & 0,84 & 1,08 & 0,89 & 18,63 & 2,34 & 17,79 \\
101 & 0,16 & 3,86 & 1,93 & 0,77 & 23,45 & 2,38 & 19,59 \\
164 & 0,17 & 3,86 & 1,89 & 1,04 & 34,16 & 2,39 & 30,30 \\
186 & 0,09 & 1,33 & 1,57 & 1,45 & 46,33 & 2,36 & 45,00 \\
207 & 0,13 & 2,22 & 1,67 & 1,60 & 56,27 & 2,31 & 54,05 \\
251 & 0,17 & 4,05 & 1,97 & 2,86 & 140,26 & 2,63 & 136,21 \\
262 & 0,16 & 3,73 & 1,90 & 4,80 & 283,57 & 2,57 & 279,84 \\
Promedio & 0,14 & 2,62 & 1,74 & -- & - & 2,37 & -- \\
\hline
\end{tabular}




\section{Discusión}

Todo el proceso de cálculo se ha basado en coeficientes y fórmulas de hidráulica general, lo cual estimamos correcto, por cuanto se trata de definir los puntos frontera en los que se inician los fenómenos torrenciales, para los cuales son válidas las formulaciones utilizadas.

Es cierto que los valores hallados no se pueden considerar, en un sentido estricto, exclusivos para cada perfil, pues no se ha considerado en los cálculos la interdependencia existente entre los perfiles por la continuidad del fenómeno de desagüe. Pero sí son valores lo suficientemente indicativos como para poder concluir que la torrencialidad que traspasa el dique quedará limitada a caudales escasamente superiores al valor de $\mathrm{Q}_{\max }$ correspondiente al perfil transversal del paramento aguas arriba del dique. $\mathrm{O}$, si se quiere ser más preciso, al perfil correspondiente a un punto del centro del cauce próximo a ese paramento, pero a una escasa distancia aguas arriba, para que podamos suponer, razonablemente, que allí las perturbaciones locales del régimen causadas por fenómenos tales como la succión o la creación de velocidad, derivadas del desagüe por los mechinales y/o por el vertedero, han desaparecido. A ese valor podemos llamar caudal máximo de torrencialidad en el dique (QTD).

Según la importancia relativa del QTD dentro del rango de caudales que se prevé que puedan circular por el cauce, se puede estudiar lo siguiente:

- Si las aguas que, transportando acarreos, acceden al tramo inferior, pueden llegar a producir depósitos o erosiones de importancia. En el caso del diqueejemplo, la torrencialidad que lo traspasaría se limita a caudales que superarán escasamente los $6,5 \mathrm{~m}^{3} / \mathrm{s}$, lo cual, en un cauce torrencial, es de un orden de magnitud pequeño, inferior incluso a muchos de los caudales generadores del lecho de los torrentes de tipo pirenáico (véase, por ejemplo, García Rodríguez, Cuchi y Gairín, 2005).

- Cuál puede ser la duración del estado transitorio de la vida útil del dique hasta su aterramiento, por cuanto el conjunto de caudales inferiores al QTD no producen depósitos en el tramo de aguas arriba (exceptuando los excesivamente gruesos). Es más: los caudales menores que QTD no sólo no colaboran en la formación del aterramiento, sino que incluso arrastrarán parte de los materiales sólidos que hayan depositado aguas más altas, en avenidas puntuales. Vol-

- viendo de nuevo al ejemplo, el conjunto de caudales inferiores a $6,5 \mathrm{~m}^{3} / \mathrm{s} \mathrm{su}$ pone un alto porcentaje de los caudales circulantes de un torrente pirenaico de pequeños recorrido y cuenca, por lo que la duración del estado transitorio puede preverse dilatada.

Otro valor interesante para la comprobación del proyecto es la distancia aguas arriba del dique a la cual pierden su carácter torrencial los caudales de mayor envergadura (superiores por lo menos a la avenida probable anual). En efecto: todo cuan- 
to se ha expuesto se basa en el supuesto de que los mechinales tienen plena capacidad de desagüe, es decir, no están obstruidos, y la principal causa de pronta obturación de los mechinales es la llegada al dique de materiales gruesos, que en principio cabe esperar sean transportados por los caudales más importantes. A partir de los resultados de nuestros cálculos podemos saber en cuál de los perfiles transversales estudiados pierden su carácter torrencial dichos caudales: si sucede a una notable distancia del dique, el depósito de los materiales gruesos no afectará en un inicio a las inmediaciones de las troneras. Decimos "en un inicio" porque debe señalarse también que este efecto será temporal: se producirá al menos en las primeras circulaciones de los caudales mayores, pero cuando se sature la capacidad de depósito del tramo variarán las condiciones del cálculo hidráulico, y los depósitos gruesos se irán aproximando progresivamente al dique. Sin embargo, no cabe duda de que es razonable esperar que el alejamiento de los materiales gruesos mantenga más tiempo despejados los mechinales, y por tanto retrase la colmatación del dique. En el caso del diqueejemplo, los caudales de más envergadura pierden su carácter torrencial a una distancia mínima del dique comprendida entre 100 y 260 metros, luego se da de manera apreciable ese alejamiento.

Otra comprobación adicional de la bondad de nuestro diseño inicial es la que resulta de comparar las velocidades iniciales de torrencialidad normal $\left(\mathrm{V}_{\text {то }}\right)$ y de torrencialidad alterada $\left(\mathrm{V}_{\mathrm{T} 1}\right)$. Si el promedio de $\mathrm{V}_{\mathrm{T} 1}$ entre los distintos perfiles resulta ser sen-

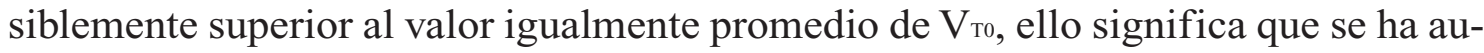
mentado notablemente la velocidad límite de arrastre en el tramo, con lo que se anulan los efectos torrenciales producidos por la circulación de la serie de caudales correspondientes a las velocidades comprendidas entre ambos valores. En el diqueejemplo, los valores promedios de $\mathrm{V}_{\text {т1 }}$ у $\mathrm{V}_{\text {то }}$ son respectivamente de $2,37 \mathrm{~m} / \mathrm{s}$ y de 1,74 $\mathrm{m} / \mathrm{s}$, lo que supone un significativo incremento del $36 \%$.

\section{Conclusiones}

- El estudio de la torrencialidad aguas arriba de un dique de corrección torrencial puede ser de gran relevancia para el adecuado diseño de este tipo de estructuras, en especial en cauces de caudal permanente.

- El cálculo de los valores de los caudales mínimo y máximo que determinan lo que se ha denominado en este comunicación "fenómeno torrencial residual" (FTR) permite comprobar en fase de proyecto si se espera del funcionamiento del dique una adecuada minimización de la torrencialidad aguas arriba, y si se transmite o no alguna influencia perturbadora aguas abajo, lo cual a su vez permite mejorar de forma iterativa el diseño de las estructuras de desagüe del dique.

— En todo caso, el diseño del dique debe ser tal que la amplitud del FTR sea el menor posible. 


\section{Bibliografía}

Blein Zarazaga, F., 1947. Utilidad de los mechinales. Montes. Publicación de los Ingenieros de Montes, 14, 157-162.

Bureau of Reclamation, 2006. Erosion and Sedimentation Manual. U.S. Department of the Interior, Denver (Colorado).

Chow, V.T., 1982. Hidráulica de los canales abiertos. Diana, Ciudad de México.

García Nájera, J.M., 1962. Principios de hidráulica torrencial. Su aplicación a la corrección de torrentes. Instituto Forestal de Investigaciones y Experiencias (IFIE). Madrid.

García Nájera, J.M.; Ripoll, D. y Jiménez, A., 1959. Hidráulica torrencial. Cálculo de diques rectos de mampostería hidráulica. Anales del Instituto Forestal de Investigaciones y Experiencias, 4, 27-36.

García Rodriguez, J.L.; Cuchí Oterino, J.A. y Gairín Muñoz, R., 2005. Revisión de los trabajos de corrección del torrente de los meses de Canfranc (Huesca). Actas $4^{\circ}$ Congreso Forestal Español, 4CFE05-301. CD-Rom. Imprenta Repes, Zaragoza.

Hager, W.M., 2010. Wastewater Hydraulics. Theory and Practice. Springer-Verlag, BerlinHeidelberg.

Jover Fernández de Bobadilla, F. y Vidania Muñoz, V., 2001. Especificaciones técnicas básicas para proyectos de corrección hidrológico-forestal de cauces torrenciales mediante hidrotecnias. Colegio de Ingenieros de Montes y TRAGSA, Madrid.

López Cadenas de Llano, F., 1965. Diques para la corrección de cursos torrenciales y métodos de cálculo. Instituto Forestal de Investigaciones y Experiencias, Madrid.

López Cadenas de Llano, F. (Dir.), 1998. Restauración hidrológico-forestal de cuencas y control de la erosión. Ministerio de Medio Ambiente, TRAGSA, TRAGASATEC y Mundi-Prensa, Madrid.

Mintegui Aguirre, J.A. y López Unzu, F., 1990. La ordenación agrohidrológica en la planificación. Servicio de Publicaciones del Gobierno Vasco, Vitoria.

Pérez-Soba Diez del Corral, I., 2002. Consideraciones sobre el cálculo por comparación de la pendiente de compensación en la corrección hidrológico-forestal. Cuad. Soc. Esp. Cienc. For., 13, 153-158.

Pérez-Soba Diez del Corral, I., 2008. Diseño y cálculo de los mechinales en diques de corrección de ramblas y torrentes. Montes. Revista de Ámbito Forestal, 93, 9-24.

Pérez-Soba Diez del Corral, I., 2011. Cálculo de la altura del vertedero de un dique de corrección torrencial, en el caso de estrechamiento súbito del perfil transversal. Cuad. Soc. Esp. Cienc. For., 32, 103-107. 
